УДК 532.593

раздел ФИЗИКА

О МОДЕЛИРОВАНИИ УДАРА ЖИДКОСТИ ПО СТЕНКЕ

\author{
() А. А. Аганин, Т. С. Гусева* \\ Институт механики и машиностроения, \\ Федеральный исследовательский иентр \\ Казанский научный иентр Российской академии наук \\ Россия, Республика Татарстан, 420111 г. Казань, ул. Лобачевского, 2/31. \\ Тел.: +7 (843) 2319114 . \\ *Email: ts.guseva@mail.ru
}

\begin{abstract}
Проведено сравнение двух известных подходов к моделированию удара жидкости по стенке. Первый основан на выполнении законов сохранения массы, импульса и полной энергии, второй, упрощенный, - на сохранении массы, импульса и энтропии. Состояние жидкости в первом подходе описывается уравнением «жесткого газа», а во втором - уравнением Тета. Сопоставлены результаты их применения для расчета начальной квазиодномерной $u$ развитой двумерной фаз первой стадии удара с присоединенной к стенке ударной волной, а также параметров, соответствующих переходу ко второй стадии с боковым растеканием. Показано, что при относительно небольших скоростях удара оба подхода дают близкие результаты. С увеличением скорости удара расхождение возрастает, причем при определенном выборе констант уравнений состояния упрощенный подход может давать более реалистичные результаты.
\end{abstract}

Ключевые слова: удар жидкости, ударные волны, критический угол, теория Хеймана.

\section{1. Введение}

Высокоскоростной удар жидкой массы (струй, капель) по поверхности тела обладает большим разрушительным потенциалом. Так, удары капель могут приводить к повреждениям паровых турбин, трубопроводных систем, летательных аппаратов в условиях дождя. Удары струй, образующихся при схлопывании кавитационных пузырьков, являются одной из причин эрозии поверхностей, контактирующих с жидкостью. Изучению удара жидкости по телу посвящено большое количество экспериментальных, аналитических и численных исследований (см., например, [1-6]). При численном моделировании удара жидкости по стенке используется как подход с применением законов сохранения массы, импульса и полной энергии, так и более простой подход на основе двух первых законов сохранения, справедливый, например, в условиях, когда среду можно считать баротропной. В данной работе для иллюстрации численного моделирования на основе этих подходов применяется метод CIP-CUP [7], в котором для перехода от одного подхода к другому требуется лишь изменение слагаемых искусственной вязкости.

Целью настоящей работы является сравнение вышеупомянутых подходов при расчете первой стадии удара жидкости по телу, характеризующейся наибольшими давлениями, а потому представляющей практический интерес. Для оценок работоспособности численных методик на этой стадии обычно используют теорию Хеймана [8], которая основана на экспериментальной зависимости скорости ударной волны от скорости потока за ее фронтом. Однако такое сравнение не всегда правомерно, поскольку совпадение или расхождение в существенной мере будет определяться тем, насколько уравнение состояния и его параметры, применяемые в численной методике, соответствуют условиям экспериментов на которых основана теория Хеймана. С учетом этого для тестирования правильности работы конкретной численной методики более корректным представляется сравнение с модификацией теории Хеймана, соответствующей принятому в этой методике подходу: с сохранением полной энергии или энтропии. Оба варианта такой модификации рассматриваются в данной работе.

\section{2. Удар жидкости по стенке}

В начале удара массы жидкости с выпуклой фронтальной частью по стенке в месте контакта возникает ударная волна, распространяющаяся вверх по бьющей жидкости (puc. 1). На первой стадии (puc. 1a) боковая граница этой волны остается присоединенной к поверхности стенки. Боковое растекание жидкости отсутствует, в сжатой жидкости, ограниченной фронтом ударной волны и стенкой, возникают очень высокие давления. Эта стадия продолжается, пока угол наклона $\theta$ поверхности бьющей жидкости к стенке не превышает некоторого критического значения $\theta_{c r}$. При $\theta>\theta_{c r}$. начинается вторая стадия (рuc. $\left.1 b\right)$, в ходе которой боковая граница ударной волны отрывается от стенки и в окрестности кромки контакта жидкости и стенки возникает радиальное растекание жидкости (пристеночная струя). При этом давление сжатой жидкости начинает снижаться. 


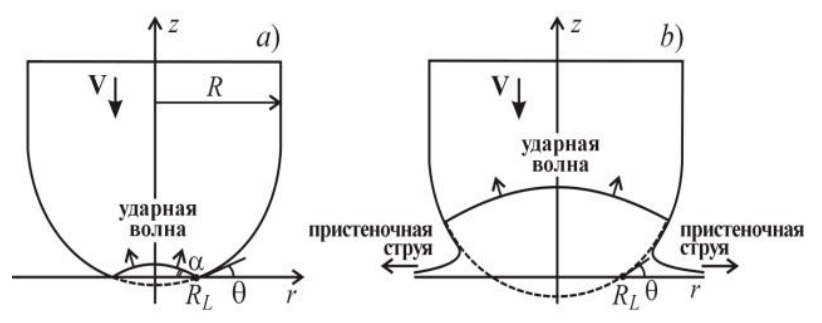

Puc. 1. Удар массы жидкости с выпуклой фронтальной частью по сухой стенке.

Таким образом, наибольшие давления на стенке возникают на первой стадии удара и сохраняются до возникновения развитого бокового растекания. При этом пространственный $\delta l$ и временной $\delta \tau$ масштабы реализации максимальных давлений на стенке очень малы. Например, в [9] для сферической капли приводятся оценки $\delta l \sim M R$ и $\delta \tau \sim M^{2} R /(2 V)$, где $V$ - скорость удара, $R$ - радиус капли, $M=V / c_{1}, c_{1}-$ невозмущенная скорость звука в жидкости. При ударе капли воды $\left(c_{1}=1500 \mathrm{M} / \mathrm{c}\right)$ при $R=1$ мм и $V=150$ м/с имеем $\delta l \sim 0.1 \mathrm{мм}, \delta \tau \sim$ 30 нс. Критический угол, при котором ударная волна отрывается от стенки, также очень мал порядка нескольких градусов [10]. Все это затрудняет как экспериментальные, так и теоретические исследования процесса удара. Полное представление о первой стадии удара имеется лишь для малых скоростей удара. Для этого случая развиты аналитические теории Лессера [11] и Коробкина [12].

\section{3. Начальная фаза первой стадии удара жидкости по стенке}

В самом начале удара воздействие на стенку близко одномерному. Давление на стенке при таких условиях характеризует давление гидроудара ("water hammer") [13]

$$
p_{\mathrm{wh}}=\rho_{1} D V
$$

где $\rho_{1}-$ невозмущенная плотность жидкости, $D-$ скорость ударной волны в случае, когда стенка ударяет со скоростью $V$ по неподвижной жидкости. Скорость $u$ жидкости за фронтом такой ударной волны равна скорости удара $V$. В работе [14] показано, что в случае воды экспериментальную зависимость $D(u)$ в широком диапазоне изменения $u$ можно с хорошей точностью аппроксимировать линейной функцией

$$
D=C+\lambda u,
$$

где $C, \lambda$ - экспериментальные константы (для воды $\lambda=2), C \approx c_{1}$.

В рамках подхода, предполагающего выполнение законов сохранения массы, импульса и полной энергии, для жидкости часто применяют уравнение состояния «жесткого газа» (например, [4])

$$
p=(\Gamma-1) \rho \varepsilon-\Gamma B,
$$

где $\varepsilon$ - удельная внутренняя энергия, Г, $B-$ экспериментальные константы (представленные ниже результаты получены для воды при $\Gamma^{\circ}={ }^{\circ} 7.15$, $\left.\mathrm{B}^{\circ}={ }^{\circ} 307.2^{\circ} \mathrm{MПа}\right)$. В таком случае давление за фронтом ударной волны определяется как

$$
p=\frac{\left[(\Gamma-1) \rho_{1}-(\Gamma+1) \rho\right] p_{1}+2 \Gamma B\left(\rho_{1}-\rho\right)}{(\Gamma-1) \rho-(\Gamma+1) \rho_{1}}
$$

При использовании соотношения (4) скорость ударной волны выражается как

$$
D=\frac{\xi}{2} u+\sqrt{\left(\frac{\xi}{2} u\right)^{2}+c_{1}^{2}},
$$

где $\xi=(\Gamma+1) / 2$.

В рамках второго подхода, не предполагающего сохранение полной энергии, используется баротропное уравнение состояния Тэта (например, [3])

$$
p=\left(p_{1}+B\right)\left(\frac{\rho}{\rho_{1}}\right)^{\Gamma}-B .
$$

Применение уравнения (б) фактически означает, что на ударной волне сохраняется лишь энтропия. В этом случае скорость ударной волны определяется неявно

$$
\left(\frac{D}{D-u}\right)^{\Gamma}-\frac{\Gamma D u}{c_{1}^{2}}-1=0 .
$$

При численном моделировании выполнение законов сохранения на фронте ударной волны обеспечивается либо за счет соответствующего построения разностной схемы, либо за счет добавления искусственной вязкости. Pис. 2 иллюстрирует результаты расчетов одномерного удара по стенке $(x=0)$ потока жидкости (воды), движущегося влево, с применением изложенной в работе [15] методики на основе метода CIP-CUP. Этот метод предполагает использование уравнений газовой динамики, записанных относительно плотности, скорости и давления. Для выполнения законов сохранения на ударных волнах применяется искусственная вязкость. При этом добавление искусственной вязкости только в уравнение для скорости (коррекция импульса) дает решение, соответствующее подходу с сохранением энтропии, a ее добавление в уравнения для скорости и давления (коррекция импульса и энергии) подходу c сохранением полной энергии. При скорости удара $50 \mathrm{~m} / \mathrm{c}$ (рuc. $2 a$ ) разница между аналитическими решениями с сохранением полной энергии и энтропии несущественна (имеет место их графическое совпадение), и с ними хорошо согласуется численное решение без добавления искусственной вязкости. При скорости удара 500 м/с (рис. 2b) аналитические решения с сохраненем полной энергии и энтропии заметно различаются. Численное решение без искусственной вязкости не согласуется ни с одним из них. Применение искусственной вязкости в соответствующих уравнениях позволяет получить хорошее согласование численных решений с соответствующими аналитическими решениями. Таким образом, в случаях, 
когда различие решений с сохранением полной энергии и энтропии существенно, при тестировании численное решение необходимо сравнивать с аналитическим решением, полученным при идентичных условиях.
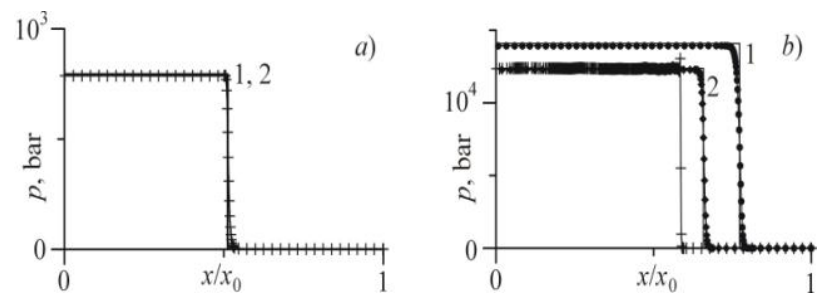

$P u c .2$. Профили давления в ударной волне, скорость удара $V=50 \mathrm{~m} / \mathrm{c}(a)$ и $500 \mathrm{~m} / \mathrm{c}(b)$. Сплошные линии без символов - аналитические решения с сохранением полной энергии (кривые 1) и энтропии (кривые 2). Кривые с символами - численные решения, рассчитанные по методике работы [15] без искусственной вязкости $(+)$, с искусственной вязкостью: в уравнении для скорости $(\diamond)$, в уравнениях для скорости и давления $(\bullet)$.

Puc. 3 иллюстрирует различие зависимостей скорости ударной волны и давления за ее фронтом от скорости за фронтом ударной волны при использовании уравнений (2), (5) и (7). Интересно, что выражение (7), соответствующее сохранению энтропии, значительно лучше аппроксимирует построенную по экспериментальным данным зависимость (2). Существенное завышение $D^{*}$ и $p^{*}{ }_{\text {wh }}$ при использовании подхода с сохранением полной энергии может быть обусловлено либо несоответствием выбранных значений констант Г и $B$ условиям эксперимента, для которого получена зависимость (2), либо некорректностью самого уравнения состояния (3) в таких условиях.
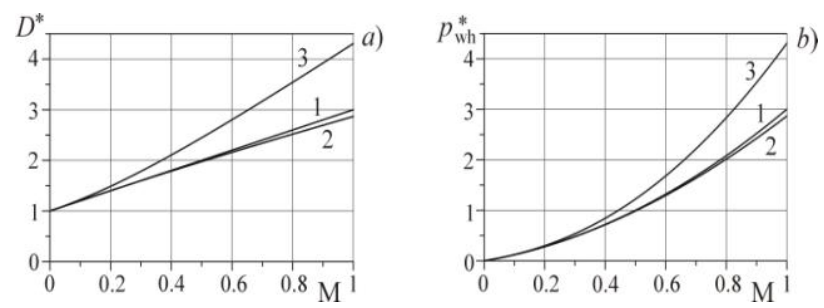

$P u c .3$. Зависимости безразмерной скорости ударной волны $D^{*}=D / c_{1}$ и безразмерного давления за ее фронтом $p^{*}{ }_{\text {wh }}=p_{\text {wh }} / \rho_{1} c_{1}{ }^{2}$ от безразмерной скорости за фронтом ударной волны $M=u / c_{1}$, соответствующие уравнениям (2) (кривые 1), (7) (кривые 2) и (5) (кривые 3).

\section{4. Развитая первая стадия удара жидкости по стенке}

В ходе первой стадии удара характер воздействия становится неодномерным: максимум давления на стенке смещается из центра области воздействия на ее периферию. Для предварительной оценки параметров жидкости на периферии области воздействия (давления, скорости и др.) обычно применяют теорию Хеймана [14] или ее модификацию Лессера [11]. В теории Хеймана предполагается, что в малой окрестности границы области контакта жидкости и стенки $r=R_{L}$ (puc. $4 a$ ) первая стадия удара развивается квазистатически. Кроме того, считается, что в каждый момент времени в этой малой окрестности ударную волну можно рассматривать как косую плоскую, которая возникает при ударе по стенке аналогичного плоского полубесконечного потока жидкости со свободной поверхностью, наклоненной к стенке под соответствующим этому моменту углом $\theta$ (puc. 4b). Возможность таких допущений подтверждается, например, в работе [10]. Для удобства анализа в теории Хеймана делается переход к подвижной системе координат $x^{*}, y$, где $x^{*}=x-V t$ $\operatorname{ctg} \theta$. Эта система связана с кромкой $x=R_{L}$ области контакта (puc. $4 b$ ), перемещающейся в ходе удара вдоль стенки со скоростью $V \operatorname{ctg} \theta$. В результате такого перехода косая ударная волна становится стационарной (рuc. 4c).

На косой стационарной ударной волне (puc. 4c) выполняются законы сохранения массы и импульса, так что

$$
\begin{aligned}
& u_{\tau 1}=u_{\tau 2}, \rho_{1} u_{n 1}=\rho_{2} u_{n 2}, \\
& p_{2}-p_{1}=\rho_{1} u_{n 1}\left(u_{n 1}-u_{n 2}\right),
\end{aligned}
$$

где $u_{n}, u_{\square}, \rho, p$ означают, соответственно, нормальную и касательную к фронту ударной волны составляющие скорости, плотность и давление, индексы 1 и 2 обозначают параметры жидкости перед и за фронтом ударной волны. Согласно обозначениям рис. $4 c$

$$
\begin{gathered}
u_{n 1}=u_{1} \sin \varphi, u_{n 2}=u_{2} \sin \alpha, u_{\tau 1}=u_{1} \cos \varphi, \\
u_{\tau 2}=u_{2} \cos \alpha, u_{1}=V / \sin \theta,
\end{gathered}
$$

где $\varphi=\alpha+\theta, \alpha-$ угол наклона ударной волны к стенке.

В теории Хеймана для замыкания соотношений (8) используется экспериментальная зависимость скорости ударной волны $D$ от скорости потока за ее фронтом (2). В принятых в уравнениях (8) обозначениях $D=u_{n 1}, u=u_{n 1}-u_{n 2}$, так что замыкающее уравнение (2) принимает вид $u_{n 1}=c_{1}+\lambda\left(u_{n 1}-u_{n 2}\right)$.
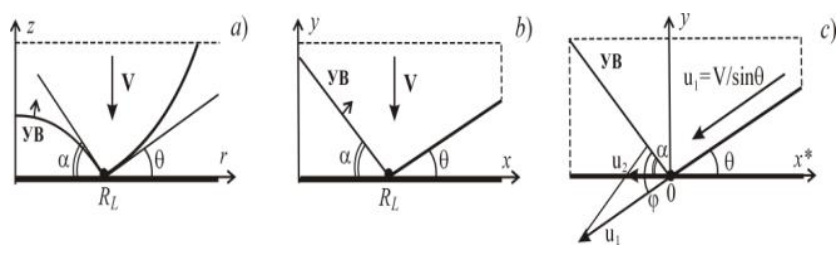

Puc. 4. Удар по стенке массы жидкости с выпуклой фронтальной частью $(a)$ и плоского потока в неподвижных $(b)$ и подвижных $(c)$ координатах.

В предложенной Лессером модификации теории Хеймана для замыкания системы (8) 
применяется уравнение Тэта (6), что соответствует изэнтропическому приближению.

Использование соотношения (4) в качестве замыкающего для системы (8) представляет собой модификацию теории Хеймана с выполнением на ударных волнах законов сохранения массы, импульса и полной энергии.

\section{5. Определение параметров за косой ударной волной}

Пусть $\bar{D}=D / c_{1}, \quad U_{1}=u_{1} / c_{1}=V /\left(c_{1} \sin \theta\right)$. Следуя [14], введем параметр $q$, характеризующий скачок плотности на фронте ударной волны, $q=1-\left(\rho_{1} / \rho_{2}\right)$. С учетом этого из уравнений $(8)$, замыкаемых (2), или (4), или (6), следует

$$
\operatorname{tg} \theta=\frac{q \bar{D} \sqrt{U_{1}^{2}-\bar{D}^{2}}}{U_{1}^{2}-q \bar{D}^{2}},
$$

где

$$
\begin{gathered}
\bar{D}=(1-\lambda q)^{-1}, \\
\bar{D}=\sqrt{\frac{1}{\Gamma q}\left[\frac{1}{(1-q)^{\Gamma}}-1\right]}, \\
\bar{D}=(1-\xi q)^{-1 / 2} .
\end{gathered}
$$

Уравнение (10) соответствует теории Хеймана и уравнению (2), уравнение (11) - ее модификации Лессера на основе уравнения Тэта (6) с сохранением энтропии, уравнение (12) - ее модификации с сохранением полной энергии с применением соотношения (4).

При заданном $\theta$ из уравнения (9) можно найти соответствующее значение $q$, после чего по одной из формул (10)-(12) рассчитать $\bar{D}$, а затем определить $\varphi$ из уравнения

$$
\operatorname{tg} \varphi=\frac{\bar{D}}{\sqrt{U_{1}^{2}-\bar{D}^{2}}} .
$$

Далее находятся другие параметры

$$
\begin{gathered}
\alpha=\varphi-\theta, p_{2}=p_{1}+\rho_{1} u_{1}^{2} \sin \varphi(\sin \varphi-\cos \varphi \operatorname{tg} \alpha), \\
u_{2}=u_{1} \frac{\cos \varphi}{\cos \alpha} .
\end{gathered}
$$

Скорость жидкости за фронтом ударной волны в неподвижной системе отсчета $\tilde{u}_{2}$ определяется выражением $\tilde{u}_{2}=-u_{2}+V \operatorname{ctg} \theta=V \operatorname{tg} \alpha$.
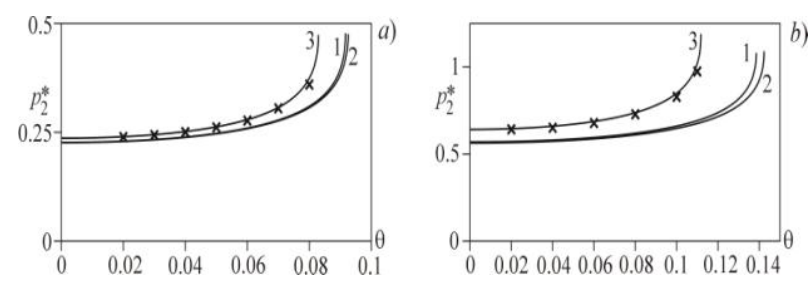

Рис. 5. Зависимости безразмерного давления за косой ударной волной $p^{*}{ }_{2}=p_{2} / \rho_{1} c_{1}{ }^{2}$ от угла $\theta$. Скорость удара $\mathrm{V}=250 \mathrm{~m} / \mathrm{c}(a)$ и $500 \mathrm{M} / \mathrm{c}(b)$. Теория Хеймана (кривые 1), модификация Лессера (кривые 2), модификация с сохранением полной энергии (кривые 3). Символы $x$ - результаты расчетов по методике работы [15] искусственной вязкостью в уравнениях для скорости и давления.

На рис. 5 приведено сравнение зависимостей безразмерного давления за косой ударной волной от угла $\theta$. Видно, что здесь, как и на рис. 3 , кривые, соответствующие модификациям теории Хеймана на основе сохранения энтропии и полной энергии, заметно расходятся. Расхождение возрастает как по мере увеличения угла $\theta$, так и с ростом скорости удара. Результаты численных расчетов существенно расходятся с теорией Хеймана, но хорошо согласуются с ее модификацией с сохранением полной энергии. Отметим, что в случае, представленном на puc. 5, модификация с сохранением энтропии дает результаты довольно близкие к теории Хеймана. Таким образом, в этом случае примение теории Хеймана вместо ее модификации с сохранением энтропии при тестировании допустимо.

\section{6. Определение критических значений параметров за косой ударной волной}

При фиксированной скорости удара $V$ конфигурация с косой ударной волной, присоединенной к стенке (puc. 4c), существует лишь при $\theta$ $\leq \theta_{c r}$. В теории Хеймана критический угол $\theta_{c r}$ принимается в качестве угла, при котором в задаче об ударе жидкой массы с выпуклой фронтальной частью завершается первая стадия удара (рuс. 1a), происходит отрыв ударной волны от стенки, и начинается вторая стадия с боковым растеканием (рuс. 1b). Согласно теории Хеймана, зависимости критических значений параметров от скорости удара $V-$ функции $\theta_{\mathrm{cr}}(M), q_{\mathrm{cr}}(M)$ и т.д., где $M=V / c_{1}$, находятся следующим образом. Сначала определяется зависимость $q_{\mathrm{cr}}\left(U_{1}\right)$ из условия

$$
\frac{\partial \operatorname{tg} \theta}{\partial q}=0 \text {. }
$$

Подстановка (9) в (15) и ряд преобразований приводят к уравнению

$$
\left(U_{1}^{2}-2 \bar{D}^{2}+q \bar{D}^{2}\right) q \bar{D}^{\prime}+\left(U_{1}^{2}-\bar{D}^{2}\right) \bar{D}=0,
$$

где скорость ударной волны $\bar{D}$ определяется одним из равенств (10)-(12), a ее производная $\bar{D}^{\prime}$, соответственно,

$$
\begin{gathered}
\bar{D}^{\prime}=\lambda \bar{D}^{2}, \\
\bar{D}^{\prime}=\frac{[(\Gamma+1) q-1] \bar{D}^{2}+1}{2 q \bar{D}(1-q)}, \\
\bar{D}^{\prime}=\xi \bar{D}^{3} / 2 .
\end{gathered}
$$

После определения и подстановки $q_{\mathrm{cr}}\left(U_{1}\right)$ в формулы (9)-(14) находятся зависимости $\bar{D}_{c r}=\bar{D}_{c r}\left(U_{1}\right), \theta_{\mathrm{cr}}\left(U_{1}\right)$ и т.д. Далее, подстановкой $\theta_{\mathrm{cr}}\left(U_{1}\right) \quad$ в $\quad$ формулу $M=U_{1} \sin \theta \quad$ находится зависимость $M_{\mathrm{cr}}\left(U_{1}\right)$. Эти функции фактически являются параметрическим определением искомых 
функций $\theta_{\mathrm{cr}}(M), q_{\mathrm{cr}}(M)$ и т.д., где роль параметра играет $U_{1}$.
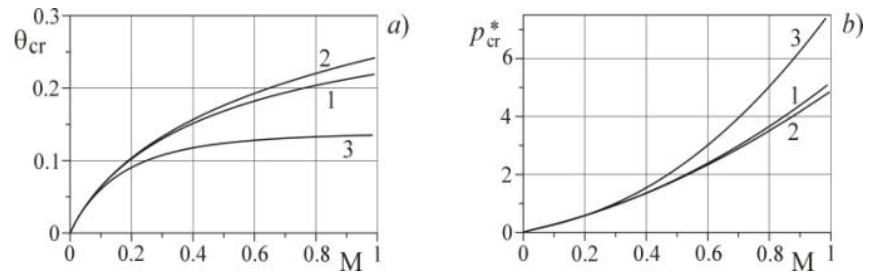

Puc. 6. Зависимости критических углов $\theta_{\text {cr }}$ и безразмерных критических давлений $p^{*}{ }_{\mathrm{cr}}=p_{\mathrm{cr}} / \rho_{1} c_{1}^{2}$ от безразмерной скорости удара $M=V / c_{1}$ по теории Хеймана (кривые 1), ее модификации Лессера (кривые 2) и модификации с сохранением полной энергии (кривые 3 ).

На рис. 6 приведено сравнение зависимостей критических параметров от скорости удара. Видно, что при относительно небольшой скорости удара все приведенные кривые близки друг к другу. По мере увеличения скорости удара расхождение возрастает. При этом, в данном частном случае кривые модификации с сохранением энтропии вновь оказываются ближе к зависимостям теории Хеймана, чем кривые модификации с сохранением полной энергии.

\section{Заключение}

Проведено сравнение двух известных подходов к моделированию удара жидкости по стенке. Первый подход предполагает применение законов сохранения массы, импульса и полной энергии в сочетании с уравнением состояния «жесткого газа». Второй подход (изэнтропический) использует лишь два первых закона сохранения в сочетании с уравнением состояния Тета. Основное внимание уделяется первой стадии удара с присоединенной к стенке ударной волной, а также параметрам, соответствующим переходу ко второй стадии с боковым растеканием. Приведены соответствующие рассматриваемым подходам модификации теории Хеймана, основанной на экспериментальных данных и часто применяемой в литературе для тестирования численных методик без обоснования корректности ее использования. Показано, что при относительно небольших скоростях удара оба подхода дают близкие результаты. С увеличением скорости удара расхождение возрастает, причем при определенном выборе констант уравнений состояния изэнтропический подход может давать результаты более близкие к оригинальной теории Хеймана, чем подход с сохранением полной энергии. Таким образом, в случаях, когда различие решений с сохранением полной энергии и энтропии существенно, при тестировании численное решение необходимо сравнивать с аналитическим решением, полученным с применением соответствующей модификации теории Хеймана.

\section{ЛИТЕРАТУРА}

1. Rein M. Phenomena of liquid drop impact on solid and liquid surfaces // Fluid dynamics Research. 1993. Vol. 12. No. 2. P. 61-93.

2. Lesser M. B. Thirty years of liquid impact research: a tutorial review // Wear. 1995. Vol. 186-187. P. 28-34

3. Чижов А. В., Шмидт А. А. Высокоскоростной удар капли о преграду // ЖТФ. 2000. Т. 170. №12. С. 18-27.

4. Haller K. K., Ventikos Y., Poulikakos D., Monkewitz P. Computational study of high-speed liquid droplet impact // J. Appl. Phys. 2002. Vol. 92. No. 5. P. 2821-2828.

5. Bourne N. K. On impacting liquid jets and drops onto polymethylmethacrylate targets // Proc. R. Soc. A. 2005. Vol. 461. P. 1129-1145.

6. Sanada T., Ando K. and Colonius T. A. Computational Study of High-Speed Droplet Impact // FDMP. 2011. Vol. 7. No. 4. P. 329-340.

7. Takizawa K., Yabe T., Tsugawa Y., Tezduyar T.E., Mizoe H. Computation of free-surface flows and fluid-object interactions with the CIP method based on adaptive meshless Soroban grids // Comput. Mech. 2007. V. 40. P. 167-183

8. Heymann F. J. High-speed impact between a liquid drop and a solid surface // J. Appl. Phys. 1969. Vol. 40. No. 13. P. 5113-5122.

9. Field J. E., Dear J. P., Ogren J. E. The effects of target compliance on liquid drop impact // J. Appl. Phys. 1989. Vol. 65. No. 2. P. 533-540.

10. Field J. E., Lesser M. B., Dear J. P. Studies of twodimensional liquid wedge impact and their relevance to liquiddrop impact problems // Proc. R. Soc. Lond. A. 1985. Vol. 401. P. 225-249.

11. Lesser M. B. Analytic solutions of liquid-drop impact problems // Proc. R. Soc. Lond. A. 1981. Vol. 377. P. 289-308.

12. Korobkin A. A. Blunt-body impact on a compressible liquid surface // J. Fluid Mech. 1992. Vol. 224. P. 437-453.

13. Cook S. S. Erosion by water-hammer // Proc. R. Soc. Lond. A. 1928. Vol. 119. P. 481-488.

14. Heymann F. J. On the Shook Wave Velocity and Impact Pressure in High-Speed Liquid-Solid Impact // J. Basic Eng. 1968. Vol. 90. No. 3. P. 400-402.

15. Аганин А. А., Гусева Т. С. Численное моделирование удара струи по стенке // Математическое моделирование. 2017. T. 29. №3. С. 123-138. 


\title{
ON THE MODELING OF WALL-LIQUID IMPACT INTERACTION
}

\author{
(C) A. A. Aganin, T. S. Guseva* \\ Institute of Mechanics and Engineering, \\ subdivision of Kazan Scientific Center of RAS \\ 2/31 Lobachevsky Street, 420111 Kazan, Republic of Tatarstan, Russia. \\ Phone: +7 (843) 2319114 . \\ *Email: ts.guseva@mail.ru
}

Two known approaches to modeling of wall-liquid impact interactions are compared. The first one is based on the mass, momentum, and total energy conservation together with the stiffened gas equation of state. The second (isentropic) one uses only the mass and momentum conservation along with the Tait equation of state. The main attention is paid to the initial quasi-1D and advanced 2D phases of the first stage of the impact with a shock wave attached to the wall, and to the parameters of the transition to the second stage with lateral liquid spreading. Two modifications of well-known Heymann theory are presented that correspond to the mentioned approaches. The theory is based on the experimental data, but it is often used for validating numerical methods without proper justification. It is shown that at relatively low impact velocity, both approaches give close results. With increase of the impact velocity, the discrepancy increases. For some constants of the equation of state, the results of the isentropic approach can be closer to Heymann theory than the results of the approach based on total energy conservation. Thus, in the cases, when the total energy and entropy conservation solutions significantly differ, the modification corresponding to the Heymann theory should be used for validating the numerical solution.

Keywords: liquid impact, shock waves, critical angle, Heymann theory.

Published in Russian. Do not hesitate to contact us at bulletin_bsu@mail.ru if you need translation of the article.

\section{REFERENCES}

1. Rein M. Fluid dynamics Research. 1993. Vol. 12. No. 2. Pp. 61-93.

2. Lesser M. B. Wear. 1995. Vol. 186-187. Pp. 28-34.

3. Chizhov A. V., Shmidt A. A. ZhTF. 2000. Vol. 170. No. 12. Pp. 18-27.

4. Haller K. K., Ventikos Y., Poulikakos D., Monkewitz P. J. Appl. Phys. 2002. Vol. 92. No. 5. Pp. $2821-2828$.

5. Bourne N. K. Proc. R. Soc. A. 2005. Vol. 461. Pp. 1129-1145.

6. Sanada T., Ando K. and Colonius T. A. FDMP. 2011. Vol. 7. No. 4. Pp. 329-340.

7. Takizawa K., Yabe T., Tsugawa Y., Tezduyar T.E., Mizoe H. Comput. Mech. 2007. Vol. 40. Pp. 167-183

8. Heymann F. J. J. Appl. Phys. 1969. Vol. 40. No. 13. Pp. 5113-5122.

9. Field J. E., Dear J. P., Ogren J. E. J. Appl. Phys. 1989. Vol. 65. No. 2. Pp. 533-540.

10. Field J. E., Lesser M. B., Dear J. P. Proc. R. Soc. Lond. A. 1985. Vol. 401. Pp. 225-249.

11. Lesser M. B. Proc. R. Soc. Lond. A. 1981. Vol. 377. Pp. 289-308.

12. Korobkin A. A. J. Fluid Mech. 1992. Vol. 224. Pp. 437-453.

13. Cook S. S. Proc. R. Soc. Lond. A. 1928. Vol. 119. Pp. 481-488.

14. Heymann F. J. J. Basic Eng. 1968. Vol. 90. No. 3. Pp. 400-402.

15. Aganin A. A., Guseva T. S. Matematicheskoe modelirovanie. 2017. Vol. 29. No. 3. Pp. 123-138. 